\title{
Actualización sobre el uso de sulfato de magnesio como neuroprotector en el parto prematuro
}

\author{
Update on the use of magnesium sulphate for fetal \\ neuroprotection in preterm birth
}

\author{
Dr. César Hernán Mellera, Dr. Gustavo Izbizky y y Dr. Lucas Otaño
}

\begin{abstract}
RESUMEN
La administración de sulfato de magnesio a la madre en riesgo de parto pretérmino como neuroprotector fetal ha demostrado reducir un 30-40\% el riesgo de parálisis cerebral y disfunción motora gruesa. Sibien existen controversias sobre el régimen de administración, la edad gestacional límite, la magnitud del potencial beneficio e, incluso, si es que hay beneficio alguno, por el momento, la evidencia es suficiente para que sea usado en pacientes en riesgo inminente de parto pretérmino antes de las 32 semanas. El objetivo del presente trabajo es mostrar la evidencia disponible y las recomendaciones actuales sobre neuroprotección con sulfato de magnesio.

Palabrasclave:sulfatodemagnesio, partopretérmino, parálisis cerebral, fármacos neuroprotectores, embarazo.
\end{abstract}

http:/ / dx.doi.org/10.5546/aap.2015.345

\section{INTRODUCCIÓN}

La parálisis cerebral es la causa más frecuente de discapacidad motora en la infancia. ${ }^{1}$ El International Executive Committee for the Definition of Cerebral Palsy propuso la siguiente definición: "Un grupo de trastornos permanentes del desarrollo del movimiento y la postura, que causan limitación de la actividad, que se atribuyen a alteraciones no progresivas ocurridas durante el desarrollo cerebral fetal o infantil. Los trastornos motores de la parálisis cerebral son acompañados a menudo por alteraciones sensoriales, perceptivas, de la cognición, comunicación y comportamiento, por epilepsia y problemas musculoesqueléticos secundarios" ${ }^{2}$

$\mathrm{Su}$ prevalencia oscila alrededor de 2-4/1000 nacidos vivos ${ }^{1,3} \mathrm{y}$, entre los principales factores de riesgo, se encuentran la prematuridad y el embarazo múltiple. La incidencia de prematuridad está en aumento; por ejemplo, en Estados Unidos, aumentó, entre 1981 y 2008 , un $36 \%$ y, en Dinamarca, entre 1995 y 2004, un 22\%. ${ }^{4}$ A su vez, el riesgo de parálisis cerebral disminuye a mayor edad gestacional al nacer: $14,6 \%$ a las $22-27$ semanas; $6,2 \%$ a las $28-31$ semanas; $0,7 \%$ a las 32-36 semanas; y $0,1 \%$ al término. ${ }^{5}$ Sin embargo, más de la mitad de los casos de parálisis cerebral se presenta en el grupo de término, dado que la mayoría de los nacimientos se producen en ese grupo. ${ }^{6}$ Un fenómeno similar se muestra con la prevalencia del embarazo múltiple, que se ha incrementado sostenidamente desde 1980 a 2009 (de 18,9 a 33,2 por 1000 nacimientos). ${ }^{7}$

Teniendo en cuenta la importancia de estos factores de riesgo, su fuerte interrelación y la posibilidad de intervenir sobre este grupo de pacientes, resulta fundamental considerar estrategias efectivas que tengan un impacto en la reducción de la tasa de parálisis cerebral. ${ }^{6} \mathrm{~A}$ continuación, se analizará la evidencia disponible y las recomendaciones de neuroprotección con sulfato de magnesio.

\section{ORÍGENES DE LA HIPÓTESIS SOBRE EL SULFATO DE MAGNESIO COMO NEUROPROTECTOR}

En el año 1995, Nelson y Grether demostraron que niños prematuros con peso menor de 1500 gramos, expuestos in utero al sulfato de magnesio (como preventivo de convulsiones eclámpticas o como tocolítico) presentaban menores tasas de parálisis cerebral, con 
una reducción del riesgo promedio mayor del 80\% (OR 0,14-IC 95\% 0,05-0,51). ${ }^{8}$ Esta y otras observaciones similares condujeron a la realización de ensayos clínicos aleatorizados con el fin específico de evaluar el rol del sulfato de magnesio como neuroprotector. Su resultado y los posteriores metaanálisis mostraron que la administración de sulfato de magnesio ante el riesgo de parto pretérmino inminente (antes de las 30-32 semanas) reducía en forma significativa el riesgo de parálisis cerebral.

\section{EFECTO BENEFICIOSO \\ DEL SULFATO DE MAGNESIO}

La revisión sistemática y metaanálisis de la colaboración Cochrane ${ }^{9}$ sobre el uso de sulfato de magnesio como neuroprotector fetal incluyó cinco estudios con 6145 niños. ${ }^{10}$ Los estudios fueron heterogéneos respecto a los criterios de inclusión (amenaza de parto pretérmino, ruptura prematura de membranas de pretérmino, preeclampsia), a la edad gestacional al momento del reclutamiento y a sus regímenes (Tabla 1).

Los principales resultados fueron los siguientes:

- Mortalidad pediátrica: No hubo diferencia significativa entre los niños expuestos y no expuestos a sulfato de magnesio: RR 1,04 (IC 95\% 0,82-1,17).

- Parálisis cerebral: La administración antenatal de sulfato de magnesio mostró una reducción relativa del $32 \%$ de parálisis cerebral: RR 0,68 (IC 95\% 0,54-0,87). El número necesario por tratar para prevenir un caso de parálisis cerebral fue de 63 (IC 95\% 39-172).

- Disfunción motora gruesa: También se evidenció una reducción significativa: RR 0,61 (IC 95\% 0,44-0,85).

La evidencia disponible hasta el momento indica que el sulfato de magnesio como neuroprotector tendría un efecto beneficioso al reducir en un $30-40 \%$ el riesgo de parálisis cerebral y disfunción motora gruesa a corto plazo. Además, recientemente, fue publicado el seguimiento a 6 años de la cohorte del estudio ACTOMgSO4 (Australasian Collaborative Trial of Magnesium-Sulphate), ${ }^{11}$ lo cual representa el seguimiento de mayor plazo reportado hasta la actualidad. ${ }^{12}$ En él, no se encontraron diferencias a nivel neurológico, cognitivo, de comportamiento, de crecimiento o funcional en la población en edad escolar entre los que habían recibido sulfato de magnesio como neuroprotector y quienes no. Resultados similares mostró el seguimiento a largo plazo del estudio francés PREMAG (Magnesium sulphate given before very-preterm birth to protect infant brain). ${ }^{13}$ Sin embargo, un aspecto que aún se desconoce es si la ausencia de beneficio a largo plazo podría deberse, por un lado, a un seguimiento incompleto de los pacientes, lo cual disminuye el poder de los estudios, y, por otro, a que el desarrollo cognitivo se encuentra influenciado por muchos factores del ambiente que no pueden ser medidos. ${ }^{14}$ Cabe destacar, además, que ninguno de los dos estudios encontró evidencia de daño en el seguimiento a largo

TABLA 1. Estudios incluidos en la revisión Cochrane con criterios de inclusión y regímenes de sulfato de magnesio.

\begin{tabular}{|c|c|c|c|}
\hline Estudio & EG al ingresar (semanas) & Intención de tratamiento & Esquema de sulfato de magnesio \\
\hline Mittendorf, $2002^{26}$ & $25-33$ & $\begin{array}{l}\text { Tocólisis (< } 4 \text { cm de dilatación cervical) } \\
\text { vs. otro tocolítico } \\
\text { Neuroprotección del feto/niño }(>4 \mathrm{~cm}) \\
\text { vs. placebo }\end{array}$ & $\begin{array}{c}\text { Bolo de } 4 \mathrm{~g}+\text { mantenimiento } \\
\text { de } 2-3 \mathrm{~g} / \mathrm{h} \\
\text { Bolo de } 4 \mathrm{~g} \text { (sin repetición) }\end{array}$ \\
\hline Rouse, $2008^{22}$ & $24-32$ & $\begin{array}{l}\text { Neuroprotección del feto/niño } \\
\text { vs. placebo }\end{array}$ & $\begin{array}{l}\text { Bolo de } 6 \mathrm{~g} \text { en } 20-30 \mathrm{~min}+ \\
\text { mantenimiento de } 2 \mathrm{~g} / \mathrm{h}\end{array}$ \\
\hline Crowther, $2003^{11}$ & $<30$ & $\begin{array}{l}\text { Neuroprotección del feto/niño } \\
\text { vs. placebo }\end{array}$ & $\begin{array}{l}\text { Bolo de } 4 \mathrm{~g} \text { en } 20 \mathrm{~min}+ \\
\text { mantenimiento de } 1 \mathrm{~g} / \mathrm{h} \\
\text { hasta } 24 \mathrm{~h} \text { o el parto }\end{array}$ \\
\hline Marret, $2008^{27}$ & $<33$ & $\begin{array}{l}\text { Neuroprotección del feto/niño } \\
\text { vs. placebo }\end{array}$ & Bolo de $4 \mathrm{~g}$ \\
\hline $\begin{array}{l}\text { Magpie Trial } \\
\text { Collaborative Group, } \\
2002^{28}\end{array}$ & $<37$ & $\begin{array}{l}\text { Neuroprotección de la madre } \\
\text { preeclámptica vs. placebo }\end{array}$ & $\begin{array}{c}\text { Bolo de } 4 \mathrm{~g} \text { en } 10 / 15 \mathrm{~min}+ \\
\text { mantenimiento de } 1 \mathrm{~g} / \mathrm{h} \text { hasta } \\
24 \text { h o de } 5 \mathrm{~g} / 4 \mathrm{~h} \text { intramuscular } \\
\text { hasta } 24 \mathrm{~h}\end{array}$ \\
\hline
\end{tabular}


plazo, lo que sugiere que los efectos beneficiosos demostrados a dos años no serían contrarrestados por efectos deletéreos posteriores. ${ }^{14}$ Sin embargo, hacen falta más estudios para alcanzar un tamaño muestral adecuado.

\section{MECANISMOS DE ACCIÓN COMO NEUROPROTECTOR}

Se cree que la parálisis cerebral sería el resultado de una lesión neuronal secundaria a un daño por inflamación, hipoxia, excitación y/u oxidación por radicales libres. ${ }^{1}$ Si bien el mecanismo exacto por el cual el sulfato de magnesio ejercería un efecto protector sobre el cerebro en desarrollo es aún desconocido, se postulan diferentes teorías: ${ }^{1}$

- Estabilidad hemodinámica.

- Prevención de la lesión por excitación y estabilización neuronal.

- Propiedades antioxidantes.

- Propiedades antiinflamatorias.

\section{INDICACIONES DEL SULFATO DE MAGNESIO COMO NEUROPROTECTOR}

La indicación del sulfato de magnesio como neuroprotector es la inminencia del parto prematuro, tanto en embarazos simples como múltiples antes de las 32 semanas. En este contexto, la inminencia del evento se define como una probabilidad elevada de que ocurra el parto por la presencia de trabajo de parto con dilatación cervical $>4 \mathrm{~cm}$, con o sin ruptura prematura de membranas, o nacimiento programado pretérmino por indicaciones maternas o fetales. ${ }^{5,15}$

Debido a la heterogeneidad entre los estudios publicados (Tabla 1), no existe consenso en relación con la edad gestacional límite para indicar sulfato de magnesio, motivo por el cual distintos países tienen protocolos diferentes. Esto se evidencia en las diversas guías publicadas. Las guías de Práctica Clínica Binacional de Australia y Nueva Zelanda ${ }^{16}$ y la del Colegio Británico de Obstetricia y Ginecología ${ }^{5}$ establecen como límite las 30 semanas, en tanto que las guías de la Sociedad de Obstetricia y Ginecología de Canadá, ${ }^{3}$ la del Colegio Americano de Obstetricia y Ginecología, ${ }^{17}$ la de la Sociedad de Medicina Materno-Fetal de Estados Unidos ${ }^{17}$ y las Recomendaciones para el manejo del embarazo y el recién nacido en los límites de la viabilidad del Ministerio de Salud de la República Argentina recomiendan hasta las 32 semanas. ${ }^{15}$

Un estudio reciente canadiense ${ }^{18}$ demostró, además, que la administración de sulfato de magnesio a embarazadas $<32$ semanas en riesgo de parto inminente sería una estrategia costoefectiva, ya que se estima que, por cada caso de parálisis cerebral prevenida, se produciría un ahorro de alrededor de 1,5 millones de dólares.

Existe controversia respecto a la costoefectividad de esta estrategia a edades gestacionales más avanzadas, dado que el riesgo de parálisis cerebral disminuye a medida que progresa la edad gestacional $y$, en consecuencia, aumenta el número necesario de mujeres por tratar para prevenir un caso de parálisis cerebral. Además, la mayoría de los estudios fueron realizados en embarazos menores de 33 semanas (Tabla 1).

\section{¿Hasta las 34 semanas?}

Si bien en Australia la neuroprotección se indica hasta la semana 30 , en la actualidad, se encuentra en curso el estudio aleatorizado multicéntrico MAGENTA -Magnesium sulphate at 30 to 34 weeks' gestational Age: Neuroprotection Trial- encabezado por la Universidad de Adelaida, que comparará la administración de sulfato de magnesio entre las 30 y las 34 semanas contra placebo. ${ }^{19}$ La hipótesis primaria es que el sulfato de magnesio administrado a las pacientes en riesgo de parto pretérmino inminente en esa edad gestacional reduce las tasas de muerte o parálisis cerebral a los dos años de edad corregida.

\section{¿Luego de las 37 semanas?}

Más de la mitad de los casos de parálisis cerebral se presenta en el grupo de niños nacidos a término. ${ }^{6,20}$ Por esto, una pregunta que surge es si el sulfato de magnesio podría tener algún rol como neuroprotector en embarazos de término con riesgo aumentado de parálisis cerebral, por ejemplo, por la presencia de restricción de crecimiento intrauterino o corioamnionitis.

Una revisión Cochrane reciente ${ }^{6}$ analizó los estudios aleatorizados en los cuales se comparó sulfato de magnesio en embarazadas a término contra placebo (un estudio con 135 pacientes con preeclampsia leve), y no se encontraron diferencias significativas en el puntaje de Apgar a los 5 minutos o la edad gestacional al momento del parto, en eventos adversos graves que forzaran la suspensión del tratamiento ni en las tasas de hemorragia posparto y cesárea.

La conclusión de los autores es que la evidencia es insuficiente aún para evaluar la eficacia y seguridad del uso de sulfato de magnesio como 
neuroprotector fetal en embarazos de término y que hace falta mayor investigación. En resumen, por ahora, no hay evidencia que avale el uso de sulfato de magnesio como neuroprotector en embarazos de término.

\section{CONTRAINDICACIONES DEL SULFATO DE MAGNESIO COMO NEUROPROTECTOR}

Las contraindicaciones para el uso de esta medicación como neuroprotector son similares que para su uso en profilaxis de eclampsia: hipersensibilidad al sulfato de magnesio, miastenia gravis, enfermedad hepática grave y malformaciones fetales o anomalías genéticas que tengan baja probabilidad de beneficio. En caso de falla renal, se debe disminuir la dosis y monitorear los niveles de magnesio.

\section{Régimen de administración}

Los regímenes utilizados para administrar el sulfato de magnesio como neuroprotector varían entre los distintos estudios (Tabla 1). Todos incluyen dosis de carga de entre 4 y 6 g, pero no todos incluyen dosis de mantenimiento. Entre los que sí lo hacen, la dosis varía entre $1 \mathrm{~g} / \mathrm{h}$ y $3 \mathrm{~g} / \mathrm{h}$. El esquema recomendado por el Ministerio de Salud de nuestro país es el siguiente: ${ }^{15}$

Dosis de carga: $4 \mathrm{~g}$ endovenosos en 30 minutos (4 ampollas de $5 \mathrm{ml} \mathrm{al} \mathrm{25 \%} \mathrm{en} 100 \mathrm{~cm}$ de dextrosa $5 \%)$.

Dosis de mantenimiento: $1 \mathrm{~g} / \mathrm{h}$ endovenoso (10 ampollas en $500 \mathrm{ml}$ de dextrosa $5 \%$ a $40 \mathrm{ml} / \mathrm{h}$ en bomba).

Debe discontinuarse la medicación en caso de que se produzca el parto, de que este deje de ser inminente o trascurridas $24 \mathrm{~h}$ del inicio de la dosis. ${ }^{15}$ Si se suspende pero posteriormente el parto vuelve a tornarse inminente, no está indicado realizar un nuevo curso de sulfato de magnesio. ${ }^{15}$

En caso de parto pretérmino programado (ejemplo: restricción de crecimiento intrauterino grave, preeclampsia grave), idealmente, se debe realizar la infusión de sulfato de magnesio, al menos, $4 \mathrm{~h}$ antes del parto. ${ }^{15} \mathrm{Un}$ análisis secundario reciente ${ }^{21}$ de un estudio multicéntrico aleatorizado ${ }^{22}$ evaluó la asociación entre duración de la infusión de sulfato de magnesio $(<12 \mathrm{~h}$, entre 12 y $18 \mathrm{~h} \mathrm{y}>18 \mathrm{~h}$ ) y resultados adversos perinatales, como muerte fetal, neonatal y parálisis cerebral, sin encontrar diferencias significativas, por lo que la duración óptima de la exposición a la medicación continúa siendo desconocida. ${ }^{21}$

Si la situación clínica demanda la finalización del embarazo en forma inmediata por motivos fetales o maternos, se recomienda no retrasar el nacimiento para realizar la infusión de sulfato de magnesio. ${ }^{15}$

\section{EFECTOS ADVERSOS}

Podemos clasificarlos en efectos adversos maternos (Tabla 2), fetales y obstétricos.

\section{Efectos adversos maternos}

El sulfato de magnesio produce vasodilatación periférica al ser utilizado en forma endovenosa. En estudios realizados con indicación de neuroprotección, los efectos más frecuentes fueron rubefacción, sudoración, náuseas y vómitos. Los

TABLA 2. Resultados maternos en estudios con uso del sulfato de magnesio como neuroprotector

\begin{tabular}{lccc}
\hline $\begin{array}{l}\text { Efectos colaterales/adversos maternos } \\
\mathbf{n}(\mathbf{\%})\end{array}$ & $\begin{array}{c}\text { Sulfato de magnesio } \\
\mathbf{n}(\mathbf{\%})\end{array}$ & $\begin{array}{c}\text { Placebo } \\
\text { (IC 95\%) }\end{array}$ & RR \\
\hline Cualquiera & $1356 / 1917(70,7)$ & $343 / 1950(17,6)$ & $5,05(2,06-12,39)$ \\
\hline Efectos menores & & & \\
$\quad$ Rubor & $1119 / 1917(58,4)$ & $614 / 1631(37,6)$ & $411 / 1631(25,2)$ \\
Molestias en el sitio de inyección & $312 / 1917(16,3)$ & $162 / 1950(8,3)$ & $68 / 1672(4,1)$ \\
Sudoración & $57 / 1672(3,4)$ & $76 / 1950(3,9)$ & $7,56(3,39-16,88)$ \\
Náuseas y vómitos & $9,12(7,19-11,57)$ & $6,37(1,96-20,68)$ & $4,60(1,54-13,75)$ \\
\hline Efectos serios & & & \\
Hipotensión & $80 / 821(9,7)$ & $56 / 535(10,5)$ & $41 / 1631(2,5)$ \\
Taquicardia & $8 / 1096(0,7)$ & $123 / 1631(7,5)$ & $0 / 1917$ \\
Depresión respiratoria & $0 / 1917$ & $52 / 805(6,5)$ & $36 / 527(6,8)$ \\
Edema pulmonar & $31 / 1672(1,9)$ & $3 / 1145(0,3)$ & $44 / 1672(2,6)$ \\
Suspensión de la infusión por efectos colaterales & $1 / 1950$ & $0 / 1950$ & $1,51(1,09-2,09)$ \\
Muerte & $1,53(1,03-2,29)$ & $1,31(0,83-2,07)$ & $2,79(0,74-10,47)$ \\
Paro cardíaco o respiratorio & $2,81(2,01-3,93)$ & $0,32(0,01-7,92)$ & - \\
\hline
\end{tabular}

RR: riesgo relativo.

Adaptado de Conde-Agudelo y Romero, 2009. 
efectos adversos maternos serios, sin embargo, fueron infrecuentes: los únicos significativos fueron hipotensión y taquicardia, y no hubo muertes maternas ni paros cardiorrespiratorios en el grupo tratado (Tabla 2). ${ }^{23}$

Un estudio aleatorizado reciente realizado en Australia (Maternal adverse effects with different loading infusion rates of antenatal magnesium sulphate for preterm fetal neuroprotection-the IRIS randomised trial) ${ }^{24}$ comparó la velocidad de administración de la dosis de carga de sulfato de magnesio como neuroprotector para evaluar si, con distintos regímenes, se podía disminuir la tasa de efectos adversos maternos. Las embarazadas fueron aleatorizadas a recibir una dosis de carga de $4 \mathrm{~g}$ de sulfato de magnesio durante 20 minutos o 60 minutos, seguida por la dosis de mantenimiento. La tasa global de efectos adversos fue del 71\% durante la primera hora, sin diferencias entre los grupos, excepto un menor número de episodios de calor y de enrojecimiento a los 20 minutos en el grupo de infusión lenta (RR 0,49, IC 95\% 0,24$0,99)$. No hubo ninguna otra diferencia, lo que incluye la suspensión de la terapia por algún evento adverso. En resumen, no pareciera que la velocidad de infusión en la dosis de carga debiera modificarse; es necesario investigar otras estrategias para disminuir la tasa de efectos adversos maternos.

\section{Efectos adversos fetales}

El uso prolongado de sulfato de magnesio (> $48 \mathrm{~h}$ ) está contraindicado debido al riesgo en fetos y neonatos de anomalías óseas y alteraciones en los niveles de calcio, fósforo y magnesio. ${ }^{20,25}$ Esto llevó a la Administración de Medicamentos y Alimentos (Food and Drug Administration; FDA, por sus siglas en inglés) a modificar la categoría para su uso en el embarazo de categoría "A" (estudios adecuados no han demostrado un riesgo para el feto) a "D" (si bien hay riesgo fetal, su uso podría aceptarse si los beneficios potenciales superan los riesgos). ${ }^{20}$ La evidencia que generó este cambio provino de estudios con graves limitaciones metodológicas para compararla con su uso como neuroprotector. La media de exposición a sulfato de magnesio en los 18 casos reportados de desmineralización y fracturas óseas fetales y neonatales fue de 9,6 semanas (67 veces la utilizada en neuroprotección), con una media de dosis materna total de $3700 \mathrm{~g}$ (157 veces la recomendada para uso obstétrico). ${ }^{25}$

El sulfato de magnesio ha sido utilizado en la práctica obstétrica probablemente desde la década de 1920, con diversas indicaciones (prevención de eclampsia, tocólisis), con décadas de farmacovigilancia, con más de 15000 mujeres enroladas y seguidas en distintos estudios aleatorizados, ninguno de los cuales ha reportado preocupación por la desmineralización o fracturas. Así, en las dosis recomendadas para alguno de sus usos obstétricos, que incluyen la neuroprotección, no es esperable que los cambios óseos representen una preocupación; la recategorización de la FDA está basada en usos no indicados y no estándares de la medicación. ${ }^{25}$

Además, estudios aleatorizados con uso de sulfato de magnesio como neuroprotector no mostraron afectar la incidencia de Apgar $<7$ a los 5 minutos (RR 1,03, IC 95\% 0,9-1,18; 3 estudios, 4387 neonatos), hipotonía neonatal (RR 1,02, IC 95\% 0,77-1,36; 1 estudio, 2444 neonatos) o la necesidad de soporte ventilatorio (RR 0,94, IC 95\% 0,89-1,00; 3 estudios, 4387 neonatos). ${ }^{3} \mathrm{~A}$ su vez, un subanálisis del estudio BEAM (Beneficial Effects of Antenatal Magnesium Sulphate Trial) no encontró correlación entre niveles de magnesio en sangre de cordón y la necesidad de ventilación, intubación o compresiones torácicas. ${ }^{3}$ Tampoco hubo diferencias en convulsiones, síndrome de distrés respiratorio, displasia brocopulmonar ni enterocolitis necrotizante. ${ }^{3}$

\section{Efectos adversos obstétricos}

En un metaanálisis del sulfato de magnesio como neuroprotector, Conde-Agudelo y Romero ${ }^{23}$ no encontraron asociación con incremento de la tasa de cesárea ( $42,9 \%$ vs. $42,8 \%$; RR 1,0, IC $95 \%$ $0,9-1,1)$ ni de hemorragia posparto grave $(3,4 \%$ vs. 263,2\%; RR 1,1 IC 95\% 0,6-1,8) cuando se comparó el grupo sulfato con el grupo placebo.

\section{MONITOREO DE LA SALUD MATERNA}

Aunque es una droga de uso frecuente en obstetricia, el uso de sulfato de magnesio requiere recaudos particulares, que incluyen el registro materno de la tensión arterial, frecuencia cardíaca, frecuencia respiratoria, reflejos tendinosos profundos (rotuliano) y diuresis. El monitoreo de la magnesemia no está indicado en forma rutinaria; solo debe controlarse en pacientes con compromiso de la función renal. ${ }^{3}$ Los eventos adversos maternos son dosis-dependientes, y el paro cardiorrespiratorio solo se alcanza con niveles de magnesemia que no se debieran alcanzar si se respetan las indicaciones y contraindicaciones de la medicación.

En caso de efectos adversos graves, se debe 
tener disponible 1 ampolla de $10 \mathrm{mg}$ de gluconato de calcio, que se administra en bolo lento.

\section{CONCLUSIÓN}

El sulfato de magnesio utilizado como neuroprotector ante la inminencia de un parto prematuro menor de 32 semanas disminuiría un $30-40 \%$ el riesgo de parálisis cerebral y la disfunción motora gruesa a corto plazo.

Si bien todavía existen controversias sobre el mecanismo de acción, régimen de administración, edad gestacional límite para su indicación, magnitud del potencial beneficio alcanzado e, incluso, si hay beneficio a largo plazo, por el momento, la evidencia pareciera ser suficiente para que el sulfato de magnesio sea usado en el manejo de las pacientes en riesgo inminente de parto pretérmino.

En primer lugar, pareciera ser efectivo sin importar cuál es la causa del parto pretérmino. Cabe aclarar que, en los estudios, prevalecieron las pacientes con trabajo de parto pretérmino y rotura prematura de membranas, y se obtuvieron similares resultados para ambas categorías. En segundo lugar, a diferencia de los corticoides, que deben ser administrados, al menos, $24 \mathrm{~h}$ antes del parto para ejercer su máximo efecto beneficioso, la ventana de oportunidad de administración del sulfato de magnesio es menor. Además, los obstetras ya están habituados al uso de esta droga, ya que se trata de los mismos regímenes utilizados en pacientes en riesgo de eclampsia.

El impacto de la estrategia de neuroprotección con sulfato de magnesio dependerá de la adherencia y cumplimiento de esta intervención por parte del equipo de atención perinatal a nivel hospitalario, que incluye obstetras, neonatólogos, obstétricas y enfermeras, en forma similar a lo que ha ocurrido con la administración de corticoides a la madre.

Actualmente, todo neonatólogo que va a recibir a un recién nacido prematuro menor de 32 semanas confirma que la madre haya recibido los corticoides. De la misma forma, su compromiso con la neuroprotección redundará en una mayor tasa de aplicación de la intervención.

\section{REFERENCIAS}

1. Costantine MM, Drever N. Antenatal Exposure to MagnesiumSulfate and Neuroprotectionin Preterm Infants. Obstet Gynecol Clin N Am 2011;38(2):351-66.

2. Rosenbaum P, Paneth N, Leviton A, Goldstein M, et al. A report: the definition and classification of cerebral palsy April 2006. Dev Med Child Neurol Suppl 2007;109:8-14.
3. Magee L, Sawchuck D, Synnes A, von Dadelszen P. SOGC Clinical Practice Guideline. Magnesium sulphate for fetal neuroprotection. J Obstet Gynaecol Can 2011;33(5):516-29.

4. Lisonkova S, Sabr Y, Butler B, Joseph K. International comparisons of preterm birth: higher rates of late preterm birth are associated with lower rates of stillbirth and neonatal death. BJOG 2012;119(13):1630-9.

5. Royal College of Obstetricians \& Gynaecologists. Magnesium sulphate to prevent cerebral palsy following preterm birth. RCOG Scientific Impact Paper 29, 2011. [Disponible en: https://www.rcog.org.uk/globalassets/ documents/guidelines/scientific-impact-papers/sip_29. pdf]. [Consulta: 19 de febrero de 2015].

6. Nguyen TM, Crowther CA, Wilkinson D, Bain E. Magnesium sulphate for women at term forneuroprotection of the fetus. Cochrane Database Syst Rev 2013;28;2:CD009395.

7. Martin JA, Hamilton BE, Osterman MJK. Three decades of twin births in the United States, 1980-2009. NCHS Data Brief 2012;(80):1-8.

8. Nelson KB, Grether JK. Can magnesium sulfate reduce the risk of cerebral palsy in very low birth weight infants? Pediatrics 1995;95(2):263-9.

9. Doyle LW, Crowther CA, Middleton P, Marret S, Rouse D. Magnesium sulphate for women at risk of preterm birth for neuroprotection of the fetus. Cochrane Database Syst Rev 2009;(1):CD004661.

10. The AMICABLE Group. Antenatal magnesium individual participant data international collaboration: assessing the benefits for babies using the best level of evidence (AMICABLE). Syst Rev 2012;1:21.

11. Crowther CA, Hiller JE, Doyle LW, Haslam RR. Effect of magnesium sulfate given for neuroprotection before preterm birth-a randomized controlled trial. JAMA 2003;290(20):2669-76.

12. Doyle LW, Anderson PJ, Haslam R, Lee KJ, et al. Schoolage outcomes of very preterm infants after antenatal treatment with magnesium sulfate vs placebo. JAMA 2014;312(11):1105-13.

13. Chollat C, Enser M, Houivet E, Provost D, et al. Schoolage outcomes following a randomized controlled trial of magnesium sulfate for neuroprotection of preterm infants. J Pediatr 2014;165(2):398-400.e3.

14. Marret S, Bénichou J. Antenatal magnesium sulfate and outcomes for school-aged children. JAMA 2015;313(3):306.

15. Argentina. Ministerio de Salud. Recomendaciones para el manejo del embarazo y el recién nacido en los límites de la viabilidad. Dirección Nacional de Maternidad e Infancia, Buenos Aires, 2014. Disponible en: http://www.msal. gov.ar/images/stories/bes/graficos/0000000513cntviabilidad.pdf. [Consulta: 19 de febrero de 2015].

16. Antenatal Magnesium Sulphate for Neuroprotection Guideline Development Panel. Antenatal magnesium sulphate prior to preterm birth for neuroprotection of the fetus, infant and child: National clinical practice guidelines. Adelaide: The University of Adelaide, 2010. Disponible en: http://www.adelaide.edu.au/arch/ antenatalMagnesiumSulphateGuidlines.pdf. [Consulta: 19 de febrero de 2015].

17. American College of Obstetricians and Gynecologists Committee on Obstetric Practice; Society for MaternalFetal Medicine. Committee Opinion No. 455: Magnesium sulfate before anticipated preterm birth for neuroprotection. Obstet Gynecol 2010 Mar;115(3):669-71.

18. Bickford C, Magee LA,Mitton C,Kruse M, etal.Magnesium sulphate for fetal neuroprotection: a cost-effectiveness analysis. BMC Health Serv Res 2013,13:527.

19. Crowther CA, Middleton PF, Wilkinson D, Ashwood P, et 
al. Magnesium sulphate at 30 to 34 weeks' gestational age: neuroprotection trial (MAGENTA)-study protocol. BMC Pregnancy Childbirth 2013;13:91.

20. Salmeen KE, Jelin AC, Thiet MP. Perinatal neuroprotection. F1000Prime Rep 2014;6:6.

21. McPherson JA, Rouse DJ, Grobman WA, Palatnik A, Stamilio DM. Association of duration of neuroprotective magnesium sulfate infusion with neonatal and maternal outcomes. Obstet Gynecol 2014;124(4):749-55.

22. Rouse DJ, Hirtz DG, Thom EA, Varner MW, et al. A Randomized trial of magnesium sulfate for the prevention of cerebral palsy. New Engl J Med 2008;359(9):895-905.

23. Conde-Agudelo A, Romero R. Antenatal magnesium sulfate for the prevention of cerebral palsy in preterm infants less than 34 weeks' gestation: a systematic review and metaanalysis. Am J Obstet Gynecol 2009 Jun;200(6):595-609.

24. Bain ES, Middleton PF, Yelland LN, Ashwood PJ, Crowther CA. Maternal adverse effects with different loading infusion rates of antenatal magnesium sulphate for preterm fetal neuroprotection: the IRIS randomised trial. BJOG 2014;121(5):595-603.

25. American College of Obstetricians and Gynecologists Committee on Obstetric Practice Society for MaternalFetal Medicine. Committee Opinion No. 573: Magnesium sulfate use in obstetrics. Obstet Gynecol 2013;122(3):727-8.

26. Mittendorf R, Dambrosia J, Pryde PG, Lee KS, et al. Association between the use of antenatal magnesium sulfate in preterm labor and adverse health outcomes in infants. Am J Obstet Gynecol 2002;186(6):1111-8.

27. Marret S, Marpeau L, Bénichou J. Benefit of magnesium sulfate given before very preterm birth to protect infant brain. Pediatrics 2008;121(1):225-6.

28. Magpie Trial Follow-Up Study Collaborative Group. The Magpie trial: a randomised trial comparing magnesium sulphate with placebo for pre-eclampsia. Outcome for children at 18 months. BJOG 2007;114(3):289-99.

Cuida tus pensamientos... se convertirán en tus palabras.

Cuida tus palabras... se convertirán en tus acciones.

Cuida tus acciones... se convertirán en tus hábitos.

Cuida tus hábitos... se convertirán en tu carácter.

Cuida tu carácter... se convertirá en tu destino.

Frank Outlaw

Quizá la ética sea una ciencia que ha desaparecido del mundo entero. No importa, tendremos que inventarla otra vez.

Jorge Luis Borges

Cuando uno se encuentra del lado de la mayoría, es hora de detenerse y reflexionar. 